\title{
Für eine Konzentration der Mittel
}

\section{Brüssel bereitet die nächste siebenjährige Förderperiode des Europäischen Sozialfonds vor}

\author{
LETITIA TÜRK \\ Letitia Türk ist wissenschaftliche \\ Mitarbeiterin im Institut für Sozial- \\ arbeit und Sozialpädagogik e. V. in \\ Frankfurt am Main und arbeitet für \\ das Projekt "Beobachtungsstelle für \\ gesellschaftspolitische Entwicklun- \\ gen in Europa«. Die Beobachtungs- \\ stelle analysiert gesellschaftspoli- \\ tische Trends in den EU-Mitglieds- \\ staaten und auf europäischer Ebene \\ sowie deren Auswirkungen auf die \\ deutsche Situation. Finanziert wird \\ das Projekt vom Bundesministerium \\ für Familie, Senioren, Frauen und \\ Jugend. \\ www.beobachtungsstelle- \\ gesellschaftspolitik.de
}

\author{
Der Europäische Sozialfonds ist das wichtigste \\ Finanzierungsinstrument der Europäischen Union \\ zur Unterstützung von Beschäftigungsmaßnahmen \\ in den Mitgliedstaaten sowie zur Förderung der \\ wirtschaftlichen und sozialen Kohäsion. Ende des \\ Jahres läuft die derzeitige Förderperiode aus.
}

Angesichts der anhaltenden Krise sind überall in Europa beträchtliche soziale und wirtschaftliche Probleme entstanden, die die Mitgliedstaaten herausfordern, wieder einen nachhaltigen Aufschwung in Gang zu bringen. Die Europäische Union und die Mitgliedstaaten stehen somit vor der Aufgabe, diesen Herausforderungen zu begegnen und gleichzeitig den in der Strategie Europa 2020 vereinbarten Zielen Rechnung zu tragen.

Die 2010 verabschiedete Strategie Europa 2020 skizziert eine »Vision für die soziale Marktwirtschaft Europas im 21. Jahrhundert ", die sich auf drei, sich gegenseitig verstärkenden Prioritäten stützt: intelligentes Wachstum Entwicklung einer auf Wissen und Innovation gestützten Wirtschaft; nachhaltiges Wachstum - Förderung einer ressourcenschonenden, ökologischeren und wettbewerbsfähigeren Wirtschaft; integratives Wachstum - Förderung einer Wirtschaft mit hoher Beschäftigung und ausgeprägtem sozialen und territorialen Zusammenhalt. (1)

Entscheidend für den Gesamterfolg bis 2020 werden diese Prioritäten sein, die in miteinander verknüpften, messbaren Kernziele ausgedrückt werden: $75 \%$ der Bevölkerung im Alter von 20 bis 64 Jahre sollen in Arbeit stehen; 3 \% des Bruttoinlandsprodukts der Europäischen Union sollen für Forschung und Entwicklung ausgegeben werden; die Klimaschutz- und Energieziele sollen erreicht werden; der Anteil der
Schulabbrecher soll auf unter $10 \%$ abgesenkt werden und mindestens $40 \%$ der jüngeren Generationen sollten einen Hochschulabschluss haben; die Zahl der armutsgefährdeten Personen sollte um 20 Millionen sinken. (2)

Eins der treibenden Instrumente zur Erreichung der sozialen Zielsetzungen im Rahmen der Europa 2020-Strategie ist die Strukturpolitik, womit die Europäische Union in die nachhaltige Entwicklung von Städten und Regionen und damit in deren Wirtschaft, Infrastruktur sowie soziale und ökologische Zukunftsfähigkeit investiert. In Vorbereitung auf die neue siebenjährige Förderperiode ab 2014 hat die sich die Europäische Kommission im Oktober 2011 auf einen Entwurf für ein Gesetzgebungspaket, das den Rahmen für die Kohäsionspolitik bilden sollte, verständigt. Insgesamt waren alle Verordnungsentwürfe auf die Ziele der Strategie Europa 2020 ausgerichtet und beinhalteten elf thematische Förderprioritäten, die ein integratives, intelligentes und nachhaltiges Wachstum unterstützen und die Bereiche Wirtschaft, Umwelt und Soziales miteinander verknüpfen sollen. (3)

Mit einem integrativen Ansatz, der in einem von der Kommission im März 2012 veröffentlichten Arbeitspapier zum Gemeinsamen Strategischen Rahmen (4) vorgestellt wurde, wurde außerdem darauf abgezielt, alle Strukturfonds aufeinander abzustimmen. Dieser Ansatz soll dazu beitragen, dass die verschiedenen Fonds auf kohärente Ziele ausgerichtet 
sind und sich so ihre Wirkung gegenseitig verstärkt. Das Budget für die Strukturpolitik wird in dem von der Kommission im Sommer 2011 vorgelegten Vorschlag für den mehrjährigen Finanzrahmen auf 376 Milliarden Euro festgelegt (vorher 347,41 Milliarden Euro), rund ein Drittel des gesamten EU-Haushaltes für den Zeitraum 2014 bis 2020. (5)

Teil dieses umfassenden Gesetzpakets für die Zukunft der Kohäsionspolitik ab 2014 war auch der Kommissionsvorschlag für den Europäischen Sozialfonds (ESF). (6) Die Rolle des Europäischen Sozialfonds besteht darin, einen Beitrag zur Entwicklung der Beschäftigung in Europa durch Förderung der Beschäftigungsfähigkeit, des Unternehmergeistes, der Anpassungsfähigkeit sowie der Chancengleichheit und der Investition in das »Humankapital« zu leisten. Somit ist der Europäische Sozialfonds das zentrale arbeitsmarktpolitische Förderinstrument der Europäischen Union.

\section{Neuausrichtung des Europäischen Sozialfonds}

Angesichts der aktuellen wachsenden Herausforderungen, wie die hohe Arbeitslosigkeit und die anhaltend hohen Armutsquoten, kommt dem Europäischen Sozialfonds eine Schlüsselrolle zu. Das betont auch die Kommission in ihrem Vorschlag zu der Ausrichtung des Europäischen Sozialfonds ab 2014, der eine Konzentration auf vier thematische Ziele vorsieht:

a) Förderung der Beschäftigung und Unterstützung der Mobilität der Arbeitskräfte

b) Förderung der sozialen Eingliederung und Bekämpfung der Armut

c) Investitionen in Bildung, Kompetenzen und lebenslanges Lernen

d) Verbesserung der institutionellen Kapazitäten und Förderung einer effizienten öffentlichen Verwaltung (7)

Darüber hinaus soll der Europäischen Sozialfonds auch zu anderen thematischen Zielen beitragen, wie: Förderung der Umstellung auf eine CO2-arme, klimaschonende und ressourceneffiziente Wirtschaft; Förderung der Nutzung von Informations- und Kommunikationstechnologien; Verstärkung von Forschung, technologischer Entwicklung und Innovation sowie Verbesserung der Wettbewerbsfähigkeit kleiner und mittlerer Unternehmen.

\section{Was ist eigentlich der Europäische Sozialfonds?}

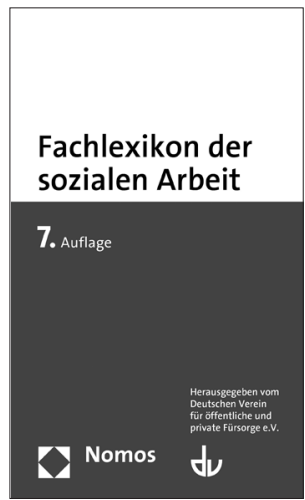

Der Europäische Sozialfonds (ESF) dient der Förderung der Beschäftigung in der Europäischen Union (EU). Er wurde bereits 1957 eingerichtet und findet seine Grundlage in Art. 162-164 des Vertrages über die Arbeitsweise der Europäischen Union (AEUV). Danach soll der ESF die berufliche Verwendbarkeit und die örtliche und berufliche Mobilität der Arbeitskräfte fördern sowie die Anpassung an die industriellen Wandlungsprozesse erleichtern, insbesondere durch berufliche Bildung und Umschulung. Der ESF ist einer der Europäischen Strukturfonds, die Unterschiede innerhalb und zwischen den Mitgliedstaaten der EU bezüglich Lebensstandard und Wohlstand verringern und so einen höheren wirtschaftlichen und sozialen Zusammenhang (Kohäsion) ermöglichen sollen. Die Mittel des ESF werden daher stärker in den wirtschaftlich schwächer entwickelten Regionen Europas eingesetzt. Der ESF unterstützt dabei die Arbeitsmarkt- und Beschäftigungspolitik in den Mitgliedstaaten. Auf europäischer Ebene wird nur ein Rahmen vorgegeben; die Mitgliedsstaaten entwickeln auf dieser Grundlage - und ergänzt durch eigene Mittel - individuelle Förderprogramme.

Der Kommissionsvorschlag sieht vor, dass der Anteil des Kohäsionsfonds im Fall des Europäischen Sozialfonds mindestens 84 Milliarden Euro betragen soll. Außerdem soll es einen Mindestanteil an Haushaltsmitteln geben, der für drei Gebietskategorien (8) zur Verfügung steht:

- mindestens $25 \%$ für weniger entwickelten Regionen

- $40 \%$ für Übergangsregionen

- $52 \%$ für stärker entwickelte Regionen

Entsprechend dem Bekenntnis der Europäischen Union $\mathrm{zu}$ integrativem Wachstum sollen mindestens $20 \%$ der ESF-Mittel für Tätigkeiten in Bezug auf
In Deutschland werden die Mittel vom Bund und von den Ländern verwaltet; es gibt daher in der Förderperiode 2007 bis 2013 insgesamt 18 Förderprogramme. Innerhalb dieser Förderprogramme wird eine Vielzahl von einzelnen Programmen mit unterschiedlicher Schwerpunktsetzung durchgeführt, z. B. zu Beschäftigung und sozialer Integration, Weiterbildung und Qualifizierung, Stärkung von Arbeitnehmer/innen und Unternehmen inkl. Existenzgründung. Auch transnationale Maßnahmen zur Stärkung des gegenseitigen Lernens werden gefördert. Für die Programme können sich bei Bund und Ländern verschiedenen Organisationen und Institutionen bewerben, die sich im Bereich Beschäftigung und soziale Eingliederung engagieren, z. B. öffentliche Verwaltungen, Wohlfahrtsverbände, Nichtregierungsorganisationen, Gewerkschaften und Arbeitgebervereinigungen. In der Förderperiode 2007 bis 2013 vergibt der ESF Mittel von ca. 75 Mrd. Euro; das entspricht rund $10 \%$ des Haushaltes der EU. Für Deutschland werden 9,38 Mrd. Euro bereitgestellt. Ca. $40 \%$ fließen dabei in das Programm des Bundes, ca. $60 \%$ in die Länderprogramme. Da im Rahmen des ESF das Prinzip der Kofinanzierung gilt - die Mitgliedstaaten müssen auch eigene Mittel einbringen -, umfasst die ESF-Förderung in dieser Zeit in Deutschland ein Finanzvolumen von insgesamt fast 16 Mrd. Euro.

Britta Spilker

Quelle: Deutscher Verein für öffentliche und private Fürsorge e. V. (Hg.): Fachlexikon der sozialen Arbeit. 7. Auflage. Nomos Verlagsgesellschaft, Baden-Baden 2011. 1.139 Seiten. 44,- Euro. ISBN 978-3-8329$5153-5$.

die soziale Eingliederung bereitgestellt werden. Auch der Bekämpfung der Jugendarbeitslosigkeit wird eine größere Bedeutung beigemessen, ebenso der Förderung von Aktivität und Gesundheit im Alter sowie der Unterstützung besonders benachteiligter Gruppen und marginalisierten Gemeinschaften wie die der Roma. Stärker gefördert werden auch Maßnahmen zur sozialen Innovation, also vor allem die Erprobung und Verbreitung neuer Praktiken zur Bewältigung sozialer Probleme.

In den operationellen Programmen, mit denen die Mitgliedstaaten den Europäischen Sozialfonds umsetzen, sollen dabei die Mittel auf eine begrenzte Zahl 
an »Investitionsprioritäten « konzentriert werden. Darüber hinaus beinhaltet der Kommissionsvorschlag spezielle Bestimmungen zum Ausbau von Partnerschaften (9) und zur Förderung der aktiven Mitwirkung von Sozialpartnern und nichtstaatliche Organisationen bei den ESF-Investitionen. Er sieht vor, dass ESF-Mittel für Kapazitätsaufbaumaßnahmen für Sozialpartner und nichtstaatliche Organisationen in weniger entwickelten Regionen bereitgestellt werden, mit dem Ziel, die Zusammenarbeit mit Sozialpartner und der Zivilgesellschaft bei der Durchführung von ESF-Tätigkeiten zu verstärken.

Der Kommissionsvorschlag vom März 2012 enthält eine Vielzahl detaillierter Grundsätze und Bestimmungen für die Programmplanung, zu Monitoring und Evaluierung sowie zur Verwaltung und Kontrolle von förderfähigen Ausgaben. Um die Effektivität der ESF-Interventionen zu verbessern, werden eine Reihe spezieller Bestimmungen vorgesehen, die für eine Konzentration der Mittel sorgen sollen. Außerdem werden gemeinsame Output- und Ergebnisindikatoren eingeführt, die ein engeres Monitoring ermöglichen und die Abschätzung der Effektivität der Investitionen auf EU-Ebene erleichtern sollen. Damit insbesondere kleineren Begünstigten die Inanspruchnahme erleichtert wird, schlägt der Verordnungsvorschlag eine Vereinfachung der Kostenerstattungsregelung vor. So sollten die Mitgliedstaaten bei kleinen Vorhaben Standardtabellen für Einheitskosten oder Pauschalbeträge anwenden, um dadurch den Verwaltungsaufwand zu reduzieren.

\section{Verhandlungsverlauf}

Auf Grundlage der Vorschläge der Europäischen Kommission hatten im März 2012 die EU-Verhandlungen zu dem mehrjährigen Finanzrahmen begonnen, zu dem auch der Europäischen Sozialfonds gehört, als Teil der Kohäsionspolitik. Allerdings war der Verlauf der Verhandlungen alles andere als problemlos. Aufgrund der von Ratspräsident Hermann van Rompuy vorgeschlagenen Kürzungen in allen Bereichen des EU-Budgets in Höhe von ca. 80 Milliarden Euro sowie einer Umverteilung der Kürzungen zwischen den Ausgabenprogrammen wurde der EUSondergipfels am 23. November 2012 ohne Einigung beendet. (10)
EU-Kommission und EU-Parlament lehnten diese Kürzungen, die 27 EURegierungen konnten sich aufgrund ihrer gegensätzlichen Interessen nicht einigen. Während den EU-Nettozahlerstaaten, darunter Deutschland, die von Ratspräsident Van Rompuy vorgeschlagenen Streichungen zu gering waren, pochten die Empfängerländer hingegen auf deutlich mehr EU-Mittel und wussten sowohl das Parlament als auch die Kommission hinter sich. (11) Weitere Quellen der Diskussion waren u. a. die geplanten Partnerschaftsvereinbarungen, die thematische Konzentration der Finanzmittel sowie die makroökonomische Konditionalität, die bestimmte Bedingungen im Zusammenhang mit der wirtschaftlichen Performanz der Mitgliedstaaten vorsieht. (12)

Erst auf dem Sondergipfel im Februar 2013 erzielten die Staats- und Regierungschefs der Europäischen Union bei der Sondersitzung des Europäischen Rates eine Einigung zu dem kommenden mehrjährigen Finanzrahmens, die weit unter dem ursprünglichen Haushaltsvorschlag der Kommission lag. Mit der vom Europäischen Rat erzielten Einigung wird die maximale Ausgabenobergrenze für die Europäische Union auf 959,9 Milliarden Euro an Mitteln für Verpflichtungen festgelegt, die Obergrenze für die gesamten Zahlungen auf 908,40 Milliarden Euro (gegenüber dem Betrag von 942,78 Milliarden Euro von 2007 bis 2013).

Für die Strukturfonds einschließlich des Europäischen Sozialfonds stehen 325,1 Milliarden Euro zur Verfügung eine Kürzung von rund $8 \%$ gegenüber dem aktuellen Finanzrahmen. Die Höhe der Strukturfondsmittel, die dem Europäischen Sozialfonds zugewiesen werden sollen, steht zurzeit allerdings noch nicht fest. Klar ist aber, dass die ESF-Mittel vorrangig zur Förderung von Wachstum und Beschäftigung eingesetzt werden sollen. So wird beispielsweise eine neue, mit ca. 6 Milliarden Euro ausgestattete Initiative zur Beschäftigung von Jugendlichen ins Leben gerufen, die zur Hälfte mit Mitteln aus dem Europäischen Sozialfonds finanziert wird. (13) Vor allem die wirtschaftlich schwächeren Regionen sollen einen größeren Anteil an Strukturfonds erhalten als bisher. Darüber hinaus soll der Europäischen Sozialfonds einen Anteil von 2,5 Milliarden Euro in der
Finanzierung eines neuen Europäischen Hilfefonds für die am stärksten von Armut betroffenen Personen übernehmen.

Damit der neue mehrjähriger Finanzrahmen im Januar 2014 in Kraft treten kann, muss erst noch eine endgültige Einigung mit dem Europäischen Parlament erzielt werden. (14) Nach den ersten Reaktionen der vier größten Fraktionen des Parlaments scheint aber der derzeitige Vorschlag der EU-Staats- und Regierungschefs im Parlament keine Mehrheit zu finden. Kritisiert werden vor allem die niedrige Gesamthöhe des Haushalts, die mangelhafte haushaltspolitische Flexibilität und die Ausgabenschwerpunkten. (15)

Somit ist derzeit ungewiss, ob es in den nächsten Monaten zu einer Einigung zwischen dem EU-Parlament und dem Rat kommen wird. Sollte das nicht passieren, wird es ab 2014 erst einmal nur jährliche Haushalte geben. Außerdem sollten die Obergrenzen des letzten Jahres aus dem vorangegangenen Finanzrahmen weiter gelten.

Ein solcher Ausgang hätte allerdings schwerwiegende Auswirkungen auf alle EU-Länder, Auswirkungen die sich momentan kaum beziffern lassen. Vor diesem Hintergrund wäre eine Lösung wünschenswert, die die finanziellen Folgen auf die Kohäsionspolitik mindert und zur Erreichung der Ziele der Strategie Europa 2020 beiträgt. Insbesondere mit Blick auf den Europäischen Sozialfonds sollte der Akzent auf seine Rolle als strategisches Investitionsinstrument zur Schaffung von wirtschaftlichem Wachstum und Beschäftigung nicht aus dem Blick geraten. In Zeiten hoher Arbeitslosigkeit und steigender Armut ist eine ausgewogene Kohäsionspolitik unabdingbar.

\section{Folgen für Deutschland}

Zurzeit werden überall in den Mitgliedstaaten die operationellen Programme (OP) für die Umsetzung der ESF-Förderperiode 2014 bis 2020 entwickelt. Auch das Bundesministerium für Arbeit und Soziales, welches für die Programme des Bundes zuständig ist, hat deren Gestaltung zu einem wichtigen Anliegen gemacht.

Um die notwendigen Förderbereiche zu identifizieren und herauszuarbeiten, hat das Ministerium die Sozialpartner, Nichtregierungsorganisationen und 
weitere Interessenten zu einem Dialog zur inhaltlichen Gestaltung der künftigen Förderung aus dem Europäischen Sozialfonds eingeladen. Interessenvertreter und Bürger erhielten die Gelegenheit, sich beispielsweise dazu zu äußern, für welche Zielgruppe sie Förderungen wünschen, welche thematische Ziele sie vorrangig gefördert sehen möchten und welche Programme fortgeführt oder modifiziert werden sollten.

Die Ergebnisse aus dem Konsultationsverfahren wurden am 21. November 2012 in einer vom Bundesministerium für Arbeit und Soziales organisierten Tagung vorgestellt. Unter den Teilnehmer der Konsultation befanden sich vor allem Trägereinrichtungen, Wohlfahrtsverbände und Vereine, Kreise und Gemeinden sowie Privatpersonen. Die Ergebnisse der Befragung zeigen, dass insbesondere die Förderung und Unterstützung von Unund Angelernten, von Personen mit Migrationshintergrund, von Jugendlichen, von Langzeitarbeitslosen und von Alleinerziehenden und Berufsrückkehrerinnen als wichtig erachtet wird.

Als nächstes werden die zukünftigen Schwerpunkte und Programme in intensiven Abstimmungen auf Bundesebene und mit den Ländern festgelegt. Der Vorschlag zum operationellen Programm des Bundes wird das Ministerium der EU-Kommission bis zum Sommer 2013 vorlegen. (16)

\section{Anmerkungen}

(1) KOM/2010/2020: Mitteilung der Kommission Europa 2020: Eine Strategie für intelligentes, nachhaltiges und integratives Wachstum.

(2) Europa 2020, S. 5.

(3) Die elf thematische Förderprioritäten sind: Stärkung der Forschung, technologischer Entwicklung und Innovation; Verbesserung des Zugangs zu sowie der Nutzung und Qualität der Kommunikations- und Informationstechnologien; Stärkung der Wettbewerbsfähigkeit von KMU, des Agrarsektors (durch den ELER) und des Fischerei- und Aquakultursektors (durch den EMFF); Förderung der Bestrebungen zur Verringerung der $\mathrm{CO} 2-$ Emmissionen in allen Branchen der Wirtschaft; Förderung der Anpassung an den Klimawandel sowie der Risikoprävention und des Risikomanagements; Umweltschutz und
Förderung der Ressourceneffizienz; Förderung der Nachhaltigkeit im Verkehr und Beseitigung von Engpässen in wichtigen Netzwerkinfrastrukturen; Förderung der sozialen Eingliederung und Bekämpfung der Armut; Investitionen in Bildung, Kompetenzen und lebenslanges Lernen; Verbesserung der institutionellen Kapazitäten und Förderung einer effizienten öffentlichen Verwaltung. KOM/2011/615 endgültig.

(4) Der Gemeinsame Strategische Rahmen ist ein Programmplanungsinstrument, mit dem die EU-Kommission die Mitgliedstaaten bei ihren Vorbereitungen für den nächsten Programmplanungszeitraum 2014 bis 2020 unterstützen möchte, vor allem bei der Formulierung von klaren Investitionsschwerpunkten. So soll der Rahmen als Ausgangspunkt für die nationalen und regionalen Behörden bei der Abfassung ihrer Partnerschaftsvereinbarungen mit der Kommission dienen.

(5) KOM/2011/500 endgültig: Mitteilung der Kommission an das Europäische Parlament, den Rat, den Europäischen Wirtschafts- und Sozialausschuss und den Ausschuss der Regionen. Ein Haushalt für »Europe $2020 \ll$.

(6) KOM/2011/607 endgültig: Vorschlag für eine Verordnung des Europäischen Parlaments und des Rates über den Europäischen Sozialfonds und zur Aufhebung der Verordnung (EG) Nr. 1081/2006.

(7) Ibid.

(8) Das neue Legislativpaket für die EUKohäsionspolitik 2014 bis 2020 sieht eine Umverteilung der Zielgebiete der Strukturfondsförderung. Statt bisher zwei soll es künftig drei Kategorien von Förderregionen geben. Diese sind. Weniger entwickelten Regionen (BIP pro Kopf unter $75 \%$ des EU-Durchschnitts); Übergangsregionen (BIP pro Kopf zwischen $75 \%$ und $90 \%$ des EU-Durchschnittes); stärker entwickelte Regionen (BIP pro Kopf über $90 \%$ des EU-Durchschnitts).

(9) Die Partnerschaftsvereinbarungen, die den Rahmen für den Einsatz der EU-Mittel bilden, sollten zwischen den EU-Mitgliedstaaten und der EUKommission abgeschlossen werden. Diese werden allerdings vor allem von den ESF-Verwaltungsbehörden kritisiert. So sprechen sich z.B. die deutschen ESF-Verwaltungsbehörden des Bundes und der Ländern dafür aus, die Vorschriften über die Partnerschaftsvereinbarung grundlegend zu überarbeiten, denn »insbesondere durch die in der Partnerschaftsvereinbarung verankerten Konditionalitäten und Sanktionsmechanismen würde der Kontroll- und Verwaltungsaufwand für die vielfältige und föderal strukturierte ESF-Förderung in Deutschland noch weiter zunehmen." In: "Zukunft des Europäischen Sozialfonds in Deutschland ab 2014 - Positionspapier der ESFVerwaltungsbehörden von Bund und Ländern zu den Legislativvorschlägen der Europäischen Kommission zur Kohäsionspolitik«.

(10) Die von dem Ratspräsident vorgelegten Verhandlungspapiere enthielten eine im Verhältnis zur Agrarpolitik überproportional höhere Kürzung bei den Strukturfonds und ebenso eine verhältnismäßig höhere Kürzung in der Kategorie »Intelligentes und integratives Wachstum «.

(11) Gegen die Kürzungen waren Bulgarien, Estland, Griechenland, Italien, Lettland, Litauen, Malta, Polen, Portugal, die Slowakei, Slowenien, Spanien, Tschechien und Ungarn. Weitere Infos auf www.tagesschau. de/wirtschaft/euhaushalt134.hhtml (28.01.2013).

(12) Entsprechend der makroökonomischen Konditionalität kann die Förderung durch EU-Kohäsionsfonds abhängig von der Erfüllung bestimmter finanzwirtschaftlicher Bedingungen gemacht und sogar ausgesetzt werden.

(13) Mit dieser Initiative sollen vor allem ESF-Initiativen in Regionen mit einer Jugendarbeitslosigkeit von über $25 \%$ durch zusätzliche Mittel für Maßnahmen unterstützt werden. http://ec.europa.eu/esf/main.jsp?ca $\mathrm{tId}=67 \&$ lang $\mathrm{Id}=\mathrm{de} \&$ newsId $=8080$ (09.02.2013).

(14) Vor der Abstimmung im Parlament müssen nun Verhandlungen mit dem Rat aufgenommen werden. Auch müssen noch ca. 75 legislative Akte, die durch den Haushaltsrahmen abgedeckt werden, formell verabschiedet werden.

(15) w w w.europarl.e uropa.eu/ news/de/headlines/content/ 
$20110429 \mathrm{FCS} 18370 / 1 / \mathrm{html} /$ EU-Haushalt-Einigung-des-Europ\% C $3 \%$ A 4 ischen-Rates-ist-nurerster-Schritt (01.03.2013).

(16) Weitere Informationen zu dem Europäischen Sozialfonds 2014-2020 in Deutschland sowie zu der Konsultationsveranstaltung zu dem Operationellen Programm des Bundes finden Sie auf der offiziellen ESF-Webseite: http://www.esf.de/portal/generator/18924/2012__11__02_konsultation.html.

\section{Literatur}

KOM/2010/2020: Mitteilung der Kommission Europa 2020: Eine Strategie für intelligentes, nachhaltiges und integratives Wachstum. KOM/2011/615 endgültig: Vorschlag für eine Verordnung des Europäischen Parlaments und des Rates mit gemeinsamen Bestimmungen über den Europäischen Fonds für regionale Entwicklung, den Europäischen Sozialfonds, den Kohäsionsfonds, den Europäischen Landwirtschaftsfonds für die Entwicklung des ländlichen Raums und den Europäischen Meeres- und Fischereifonds, für die der Gemeinsame Strategische Rahmen gilt, sowie mit allgemeinen Bestimmungen über den Europäischen Fonds für regionale Entwicklung, den Europäischen Sozialfonds und den Kohäsionsfonds und zur Aufhebung der Verordnung (EG) Nr. 1083/2006. KOM/2011/500 endgültig: Mitteilung der Kommission an das Europäische Parlament, den Rat, den Europäischen Wirtschafts- und Sozialausschuss und den Ausschuss der Regionen. Ein Haushalt für „Europe 2020“.

KOM/2011/607 endgültig: Vorschlag für eine Verordnung des Europäischen Parlaments und des Rates über den Europäischen Sozialfonds und zur Aufhebung der Verordnung (EG) Nr. 1081/2006.

\section{„Zukunft des Europäischen Sozialfonds in} Deutschland ab 2014 - Positionspapier der ESFVerwaltungsbehörden von Bund und Ländern zu den Legislativvorschlägen der Europäischen Kommission zur Kohäsionspolitik“.

\section{Offizielle Webseite Europäischer Sozialfonds} Deutschland: http://www.esf.de/portal/generator/18924/2012__11__o2__konsultation.html Offizielle Webseite des Europäischen Parlaments: http://www.europarl.europa.eu/news/ de/headlines/content/20110429FCS18370/1/ html/EU-Haushalt-Einigung-des-

Europ\%C3\%A4ischen-Rates-ist-nur-erster-Schritt Offizielle Webseite der Europäischen Kommission zu dem Europäischen Sozialfonds: http:// ec.europa.eu/esf/home.jsp?langld=de

\section{MITDENKEN MITGESTALTEN}

Die Bertelsmann Stiftung ist eine der größten operativen Stiftungen in Europa. Sie ist eine gemeinnützige Organisation, die sich in der Tradition ihres Gründers Reinhard Mohn für eine zukunftsfähige Gesellschaft engagiert. Mit unserem Programm Zukunft der Zivilgesellschaft geben wir Impulse, die Menschen vermehrt zum Engagement und zum sozialen Investment motivieren und die zivilgesellschaftliche, staatliche und privatwirtschaftliche Organisationen zu langfristigen Kooperationen und gemeinsamem Wirken anregen.

Das Projekt "Social Investment" unterstützt den Aufbau eines Marktes für soziale Investitionen in Deutschland. Für dieses Projekt suchen wir ab sofort einen

\section{Projektmanager $(\mathrm{m} / \mathrm{w})$ „Social Investment"}

\section{Ihre Aufgaben}

Gemeinsam mit dem Projektteam und deutschen sowie internationalen Partnern erstellen Sie Analysen zu Leistungsbereichen der Sozialwirtschaft und entwickeln Instrumente zu Wirkungsanalysen. Sie ermitteln den Finanzbedarf in ausgewählten Feldern und erstellen Machbarkeitsstudien zu unterschiedlichen Investitionsformen. Daneben arbeiten Sie an der Konzeption und Realisierung von Veranstaltungen und Veröffentlichungen mit.

\section{Unsere Anforderungen}

- Erfolgreich abgeschlossenes Studium

- Fundiertes Wissen zu zivilgesellschaftlichen Fragestellungen

- Praktische Berufserfahrungen in der Sozialwirtschaft

- Kenntnisse zu Wirkungsanalysen

- Erfahrung mit strategischem Partnermanagement und Netzwerken

- Analytische Fähigkeiten und ganzheitliches Denken

- Hohe Team- und Kooperationsfähigkeit

- Eigeninitiative und ergebnisorientiertes Handeln

- Bereitschaft zu umfangreicher Reisetätigkeit

- Exzellente, verhandlungssichere Kenntnisse der englischen Sprache in Wort und Schrift

Sie können in der Bertelsmann Stiftung eine verantwortungsvolle Aufgabe in einem aufgeschlossenen Team erwarten. Wir freuen uns auf Sie!

Bitte senden Sie Ihre Bewerbung inklusive Ihrer Gehaltsvorstellung sowie dem frühestmöglichen Eintrittstermin an Petra Johannkemper, Personalabteilung Bertelsmann Stiftung, Carl-Bertelsmann-Straße 256, 33311 Gütersloh. Bitte nutzen Sie für Ihre Bewerbung bevorzugt unser Online-Bewerbungsformular unter www.karriere. bertelsmann-stiftung.de oder schicken Sie Ihre Bewerbung per E-Mail an stiftung@ bertelsmann.de.

www.bertelsmann-stiftung.de/karriere 